\title{
Comunicación
}

\section{Tumor de células de Sertoli en un canino macho sin criptorquidismo: reporte de caso}

\author{
Sertoli cells tumor in a male canine without cryptorchidism: case report \\ Orly Damian Gutierrez', German Jaramillo Rodríguez ${ }^{1}$, \\ Jhonny Alberto Buitrago Mejia ${ }^{1,2}$
}

\section{Resumen}

Las neoplasias testiculares son un diagnóstico diferencial para considerar en la evaluación clínica de perros que se llevan a consulta por enfermedades del tracto genital. Se consideran patologías de fácil de diagnóstico, aunque su confirmación precisa del estudio histopatológico, siendo su presentación más frecuente en gerontes y criptorquidos. La orquiectomía bilateral es la terapéutica de elección, siendo curativa en la mayoría de los casos. Se presenta el caso de un canino mestizo de cinco años, macho entero no criptorquido. El propietario reporta un aumento de tamaño en la región inguinal de crecimiento continuo con un mes de evolución en la región inguinal izquierda. Se realiza ecografía e histopatología como ayudas diagnósticas determinándose la presencia de un tumor de células de Sertoli.

Palabras clave: tumor de células de Sertoli; canino; ecografía; histopatología

\section{AbSTRaCt}

Testicular neoplasms are a differential diagnosis to consider in the clinical evaluation of dogs that consult for diseases of the genital tract. Its presentation is more frequent in old and cryptorchid dogs. They are considered pathologies of easy diagnosis, although their precise confirmation of the histopathological study. Bilateral orchiectomy is the therapeutic of choice, being curative in most cases. The case of a five-year-old crossbred

\footnotetext{
${ }^{1}$ Grupo de Investigación GINVER, Programa de Medicina Veterinaria, Facultad de Medicina Veterinaria, Medellín, Corporación Universitaria Remington, Colombia

${ }^{2}$ E-mail: jhonny.buitrago@uniremington.edu.co
}

Recibido: 11 de agosto de 2018

Aceptado para publicación: 5 de septiembre de 2019 
canine, intact male, not cryptorchid is presented. The owner reports an increase in size in the inguinal region of continuous growth with a month of evolution in the left inguinal region. Ultrasound and histopathology are performed as diagnostic aids and the presence of a Sertoli cell tumour was found.

Key words: Sertoli cell tumor; canine; ultrasound; histopathology

\section{INTRODUCCIÓN}

Las neoplasias testiculares son poco frecuentes en los animales domésticos, siendo reportados con mayor frecuencia en la clínica de la especie canina, en donde generalmente pasan desapercibidas para los propietarios y son hallazgos de la exploración física (Ortega y Avalos, 2000; Martí et al., 2010). Se estima que estas patologías corresponden al $0.7-10 \%$ de todos los tumores diagnosticados en caninos (Eslava y Torres, 2008; D'Angelo et al., 2012; Švara et al., 2014), y a cerca del $90 \%$ de las neoplasias genitales en perros machos (D'Angelo et al., 2012). Su presentación es más frecuente en animales geriatras y criptórquidos. Se consideran patologías de fácil de diagnóstico, aunque su confirmación precisa del estudio histopatológico. En caninos, la orquiectomía bilateral es la terapéutica de elección, siendo curativa en la mayoría de los casos (Ortega y Avalos, 2000; Martí et al., 2010; Ano et al., 2014; Vieira et al., 2016).

Las neoplasias testiculares más comunes en perros son el tumor de células de Sertoli, el tumor de las células de Leydig y el seminoma ((Nødtvedt et al., 2010; Domingos y Salomão, 2011; Švara et al., 2014). Estas neoplasias se consideran de baja malignidad, siendo poco frecuentes las metástasis. Las presencias de testículos ectópicos favorecen el desarrollo de seminomas y tumores de células de Sertoli (Martí et al., 2010; Domingos y Salomão, 2011; Quartuccio et al., 2012; Vieira et al., 2016), pudiendo coexistir más de un tipo de tumor en un mismo individuo (Vieira et al., 2016). En el presente re- porte se describe un caso de tumor de células de Sertoli en un canino no criptorquido.

\section{Evaluación del Paciente}

\section{Anamnesis}

Llega a consulta un canino mestizo, macho entero de cinco años. El propietario reporta un aumento de amaño en la región inguinal de crecimiento continuo con un mes de evolución. Adicionalmente reporta que se desplaza con tres extremidades y solo apoya los cuatro miembros al correr.

\section{Examen Físico}

En la evaluación clínica se encuentran los signos vitales dentro de los parámetros normales, peso de $13.5 \mathrm{~kg}$ y de buena condición corporal. Como hallazgo relevante se observa una masa de aproximadamente 15

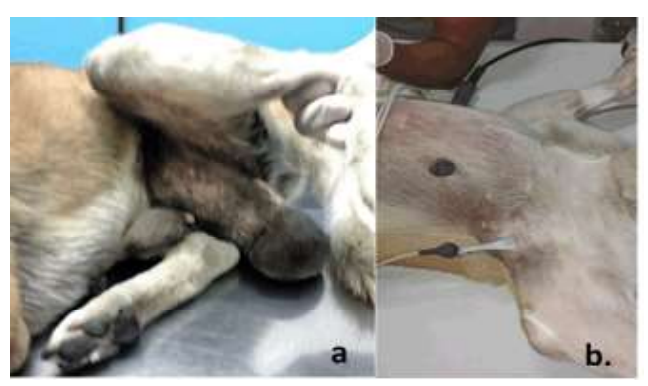

Figura 1. Perro mestizo de 5 años con tumor de células de Sertoli. (a) Masa pendulante de $15 \mathrm{~cm}$ en la región inguinal izquierda; (b) Ginecomastia e hiperpigmentación región torácica 
Cuadro 1. Hemograma pre-quirúrgico en perro mestizo de cinco años con una masa tumoral de15 cm de longitud en la región inguinal, involucrando la bolsa escrotal izquierda

\begin{tabular}{lcc}
\hline Analito & Resultado & Valor de referencia $^{1}$ \\
\hline Glóbulos blancos $\left(\mathrm{x} 10^{3} / \mu \mathrm{l}\right)$ & 12.4 & $6.0-17.0$ \\
Glóbulos rojos $\left(\mathrm{x} 10^{6} / \mu \mathrm{l}\right)$ & 6.10 & $5.5-8.5$ \\
Hemoglobina $(\mathrm{g} / \mathrm{dl})$ & 14.2 & $12-18$ \\
Hematocrito $(\%)$ & 42.5 & $37-55$ \\
MCV $(\mathrm{fl})$ & 69.7 & $60-77$ \\
MCH $(\mathrm{pg})$ & 23.2 & $20-25$ \\
$\mathrm{MCHC}(\mathrm{g} / \mathrm{dl})$ & 33.3 & $32-36$ \\
I.D.E $(\%)$ & 14.4 & $12-15$ \\
Plaquetas $\left(\mathrm{x} 10^{3} / \mu \mathrm{l}\right)$ & 390 & $200-500$ \\
Neutrófilos $\left(\mathrm{x} 10^{3} / \mu \mathrm{l}\right)$ & 8.7 & $3-77.5$ \\
Linfocitos $\left(\mathrm{x} 10^{3} / \mu \mathrm{l}\right)$ & 2.9 & $1-4.8$ \\
Eosinófilos $\left(\mathrm{x} 10^{3} / \mu \mathrm{l}\right)$ & 0.1 & $0.1-1.25$ \\
Monocitos $\left(\mathrm{x} 10^{3} / \mu \mathrm{l}\right)$ & 0.6 & $0.15-1.35$ \\
Bandas $\left(\mathrm{x} 10^{3} / \mu \mathrm{l}\right)$ & 0.1 & $0-0.3$ \\
Proteínas totales $(\mathrm{g} / \mathrm{dl})$ & 7.9 & $6-8$ \\
Creatinina $(\mathrm{mg} / \mathrm{dl})$ & 0.82 & $0.5-1.5$ \\
ALT $(\mathrm{UI} / \mathrm{L})$ & 21 & $21-102$ \\
\hline
\end{tabular}

MCV: Volumen corpuscular medio; MCH: Hemoglobina corpuscular media; VCM: Concentración de hemoglobina corpuscular media (CHCM); ALT: Alanina aminotransferasa

${ }^{1}$ Valores reportados por el laboratorio clínico de referencia (Test Laboratorio SAS, Medellín, Colombia)

cm de longitud al lado izquierdo de la región inguinal, involucrando la bolsa escrotal izquierda (Figura 1a), de consistencia semidura y dolorosa a la palpación. Así mismo, se observa en la zona torácica una tendencia a la ginecomastia con hiperpigmentación cutánea (Figura 1b).

En el examen hemático (Cuadro 1) no se encuentran alteraciones clínicas relevantes. En el examen ecográfico (Mindray M5 Vet, con sonda microconvexa de $7.5 \mathrm{MHz}$; Figura 2) se reporta la presencia de una masa en el espacio subcutáneo de la región inguinal izquierda que continua hacia el saco escrotal con ecotextura fina y heterogénea de aproximadamente $15 \times 10 \mathrm{~cm}$; asimismo, aumento de tamaño prostático con estructuras quísticas intraprostáticas simples y aumento de linfonodos iliacos.
Se realiza la orquiectomía mediante abordaje quirúrgico escrotal y se encuentra un testículo izquierdo de $450 \mathrm{~g}$ y $15 \mathrm{~cm}$ de longitud, con abundante irrigación sanguínea (Figura 3a). Se realiza histopatologia de varias porciones del tejido testicular afectado.

\section{Examen Histopatológico}

Se observa una proliferacion neoplásica de células poligonales que se organizan mediante extensos agregados (Figura 3b), obliterando el lumen de los túbulos. Las celulas neoplásicas presentan nucleo redondeado a oval con anisocariosis leve a moderada, cromatina fina, nucleolo notorio (uno por núcleo) y citoplasma eosinófilo de límites indistintos. Las mitosis son escasas. Se percibe atrofia severa del epitelio germinal espermático (azoospermia). Se diagnostica como tumor de células de Sertoli. 

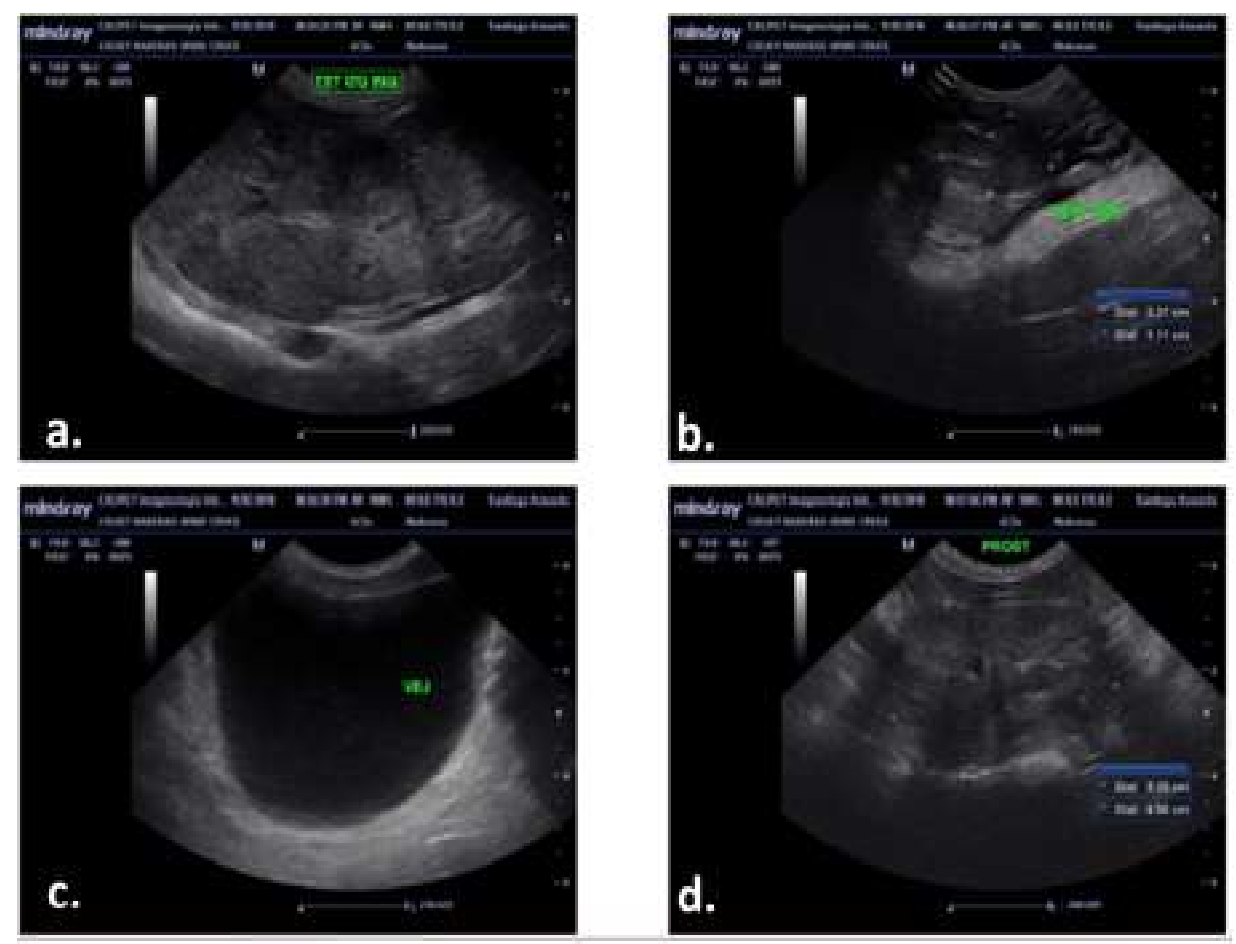

Figura 2. Ecografía testicular y prostática de perro mestizo de cinco años. (a) Testículo izquierdo aumentado de tamaño, ecogenicidad heterogénea y múltiples zonas anecoicas; (b) Testículo derecho presenta una leve reducción de tamaño $(2.2 \times 1.5 \mathrm{~cm})$, pero conserva su ecogenicidad normal; (c) La vejiga no presenta hallazgos anormales; (d) La próstata se encuentra aumentada de tamaño $(3.2 \times 4.2 \mathrm{~cm})$, con ecogenicidad heterogénea y zonas anecoicas bien delimitadas en su interior compatibles con lesiones quísticas

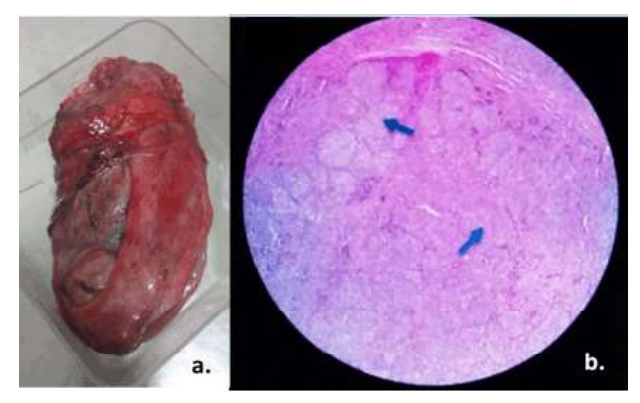

Figura 3. (a) Masa exraida del escroto de un perro mestizo de cinco años que corresponde a testiculo izquierdo; (b) Parénquima testicular. 100x. Se observa proliferación neoplásica de células poligonales intratubulares que se organizan mediante agregados (flechas azules)

\section{Discusión}

Los tumores de células de Sertoli generalmente se observan en perros entre los 8 y 11 años. El riesgo de desarrollar este tipo de tumor puede aumentar hasta 14 veces cuando los testículos están retenidos en la cavidad abdominal (Ortega y Avalos, 2000), de allí que la mayoría de reportes de este tipo de casos sean de animales criptórquidos (Eslava y Torres, 2008; Hong et al., 2011; D'Angelo et al., 2012; Quartuccio et al., 2012; Vieira et al., 2016; Madureira et al., 2017), lo que hace interesante este caso, pues se trata de un paciente de talla mediana de cinco años sin criptorquidia. 
Dentro de los signos más característicos del tumor de células de Sertoli se encuentran la feminización, pérdida de libido, ginecomastia, alopecia y atrofia del testículo no afectado (Ortega y Avalos, 2000; Planellas et al, 2007), debido al hiperestrogenismo, por lo que el diagnóstico de este tipo de tumor requiere la determinación de estrógenos en sangre (Ano et al., 2014). Sin embargo, los laboratorios suelen medir solamente $17 \beta$ estradiol, mientras que los perros tienen además estrógenos circulantes bajo la forma de estrona y estriol, pudiendo de esa forma encontrase casos de perros con niveles normales de $17 \beta$-estradiol (Sanpera et al., 2002). También se ha explorado la determinación de los niveles de hormona antimulleriana (AMH) mediante técnicas de ELISA para el diagnóstico de esta patología, ya que parece que esta se encuentra elevada en pacientes con esta patología (Banco et al., 2012; Ano et al., 2014)

En este caso clínico se presentó una forma leve del síndrome feminizante, que se reporta como característico de la enfermedad y, a menudo, como el único signo clínico externo de este cambio neoplásico. Asociado a este síndrome también se han descrito disfunciones prostáticas que incluyen hiperplasia benigna, metaplasia escamosa y quistes prostáticos con síntomas relacionados (Quartuccio et al., 2012), tal como ocurrió en este caso.

La evaluación ecográfica es el método de imagen más indicado para la evaluación de los tumores testiculares (Domingos y Salomão, 2011). En la ecografía del tumor de células de Sertoli es frecuente encontrar el testículo afectado con aumento de tamaño, parénquima heterogéneo y el testículo contralateral disminuido de tamaño o de dimensiones normales con parénquima homogéneo (Vieira et al., 2016); sin embargo, ecográficamente no se puede diferenciar de otros tipos de tumores testiculares y, por lo tanto, se requiere de una biopsia del testículo afectado para realizar el estudio histopatológico para el diagnóstico definitivo (Martí et al., 2010; Cahua y Sandoval, 2015).
La orquiectomía bilateral es la terapéutica de elección en el caso de las neoplasias testiculares (Vieira et al., 2016), aunque también se han reportado otras alternativas de tratamiento como la quimioterapia, que pueden dar resultados positivos en la regresión de las células tumorales hasta en un $50 \%$ del tamaño inicial, pero los altos costos y lo prolongado del tratamiento hacen inviable esta alternativa (Eslava y Torres, 2008). Finalmente, es necesario considerar que si bien es una patología benigna existe la posibilidad de metástasis funcionales (Navarrete et al., 2015)

\section{Literatura Citada}

1. Ano H, Hidaka Y, Katamoto H. 2014. Evaluation of anti-Müllerian hormone in a dog with a Sertoli cell tumour. Vet Dermatol 25: 142-145. doi: 10.1111/ vde. 12112

2. Banco B, Veronesi MC, Giudice C, Rota A, Grieco V. 2012. Immunohistochemical evaluation of the expression of anti-Müllerian hormone in mature, immature and neoplastic canine Sertoli cells. J Comp Pathol 146: 18-23. doi: 10.1016/j.jcpa.2011.03.011

3. Cahua J, Sandoval N. 2015. Piometra y tumor de Sertoli en un canino con conducto de Müller persistente. Rev Inv Vet Perú 26: 705-711. doi: 10.15381/ rivep.v26i4.11208

4. D'Angelo AR, Vita S, Marruchella G, Di Francesco G.2012. Canine testicular tumours: a retrospective investigation in Abruzzo and Molise, Italy. Vet Ital 48: 329-333.

5. Domingos TCS, Salomão MC. 2011. Meios de diagnóstico das principais afecções testiculares em cães: revisão de literatura. Rev Bras Reprod Anim 35: 393-399. 
6. Eslava MP, Torres VG. 2008. Neoplasias testiculares en caninos: un caso de tumor de células de sertoli. Rev MVZ Córdoba 13: 1215-1225.

7. Hong S, Lee HA, Han SJ, Kim O. 2011. Spontaneous Sertoli cell tumor with cryptorchism in a beagle dog. Lab Anim Res 27: 177-178. doi: 10.5625/ lar.2011.27.2.177

8. Madureira R, De Paula-Reis N, Rossotti dos Santos A, Dias AL, Arlington Headley $S$, Freres Mascarenhas NM, Sperotto Brum J. 2017. Seminoma and sertolioma in a male pseudohermaphrodite dog with unilateral cryptorchidism: short communication. Semin-Cienc Agrar 38: 28312836. doi: $10.5433 / 1679-0359.2017 \mathrm{v}-$ 38n4Supl1p2831

9. Martí S, Cloquell A, Vázquez F, Díaz $A .2010$. Canine testicular tumours: two clinical cases report. Clin Vet Peq Anim 30: 191-198.

10. Navarrete-Méndez, RodríguezHuitrón A, Hernández-Ballesteros J, Benítez-Meza A, Orozco-Benítez G. 2015. Testicle tumors in the dog. Abanico Vet 5: 49-57.

11. Nodtvedt A, Gamlem H, Gunnes G, Grotmol T, Indrebo A, Moe L. 2010. Breed differences in the proportional morbidity of testicular tumours and distribution of histopathologic types in a population-based canine cancer registry.
Vet Comp Oncol 9: 45-54. doi: 10.1111/ j.1476-5829.2010.00231.x

12. Ortega-Pacheco A, Avalos-Borges EE 2000. Hiperestrogenismo, alopecía y metaplasia escamosa de próstata asociados a un tumor de células de Sertoli en un perro. Rev Biomed 11: 33-38.

13. Planellas M, Martínez I, Peña M, Pastor J. 2007. Síndrome de feminización en un perro con un tumor testicular de células de Sertoli. Clin Vet Peq Anim 27: 109-113.

14. Quartuccio M, Marino G, Garufi G, Cristarella S, Zanghì A. 2012. Sertoli cell tumors associated with feminizing syndrome and spermatic cord torsion in two cryptorchid dogs. J Vet Sci 13: 207209. doi: 10.4142/jvs.2012.13.2.207

15. Sanpera N, Masot N, Janer M, Romeo C, De Pedro R. 2002. Oestrogeninduced bone marrow aplasia in a dog with a Sertoli cell tumour. J Small Anim Pract 43: 365-369. doi: 10.4142/ jvs.2012.13.2.207

16. Švara T, Gombaè M, Pogorevc E, Plavec T, Zrimšek P, Pogaènik. 2014. A retrospective study of canine testicular tumours in Slovenia. Slov Vet Res 51: 81-88. doi: 10.13140/2.1.1966.9123

17. Vieira F, Ferreira FN, de Andrade M, dos Santos R. 2016. Tumor de células de Sertoli e seminoma difuso em cão com criptorquidismo bilateral - relato de caso. Rev Bras Med Vet 38: 217-221. 